\title{
Hydroxychloroquine; Why It Might Be Successful and Why It Might Not Be Successful in The Treatment of Covid-19 Pneumonia? Could It Be A Prophylactic Drug?
}

Omer Deniz, MD

Pulmonologist

Professor in Pulmonary Medicine,

Burc Mahallesi, Yenimahalle, Ankara, Turkey

E-mail: omerdeniz@yahoo.com

\section{Introduction}

Even though there is no double blind randomized placebocontrolled studies, according to current literature and preliminary data, mainly composed of cell culture, animal and smalluncontrolled human studies, hydroxychloroquine (HCQ) seems potentially effective in the treatment of the patients with Covid-19 pneumonia ${ }^{1-5}$. It is very well known that, in normal conditions, no drug can be approved for the treatment of human-beings based on the evidence originating only from cell cultures, animal studies or uncontrolled small studies with the absence of phase I, II, III drug studies. Since HCQ is an old drug and repurposed for treatment of Covid-19 disease, phase II studies might not be necessary. Further more, it is almost impossible to determine an exact drug dosage to clear the virus from the body based on the animal or cell culture studies $^{3,6,7}$. However, since there is an urgent pandemic situation, even in the absence of sufficient scientific evidence, the role of HCQ in the treatment of Covid-19 pneumonia is debated among many experts. Some experts advocate to use HCQ with some precautions or case by case, some advocate waiting for the results of ongoing 
HCQ-Covid-19 trials, some find its use potentially harmful ${ }^{8,9}$. On the other hand, in practice, HCQ is commonly used for the treatment of Covid-19 disease worldwide. Its use is recommended in interim guidelines of Turkey ${ }^{10}$ and Belgium ${ }^{11}$ for the treatment of Covid-19 disease. It is recommended as a prophylactic drug in persons with high-risk for developing Covid-19 disease in India ${ }^{12}$.

\section{Mechanism of Action and Pharmacokinetics of HCQ}

HCQ is believed to show its antiviral activity against SARSCoV-2 virus at least by two ways, first by interfering with the glycosylation of ACE2 receptor, thus reducing the ability of the SARS-CoV-2 virus to bind on the ACE2 receptors existing on the surface of the host cells, second increasing the endosomal $\mathrm{pH}$ which blocks further stages of viral replication ${ }^{1,2,7,9}$. In addition, since HCQ might be a zinc ionophore and zinc might have some antiviral effects it might also be hypothesized that addition of zinc to HCQ therapy might be more effective than HCQ alone ${ }^{13-15}$. However, data about HCQ plus zinc therapy for Covid-19 disease is very limited for now.

HCQ has a large volume of distribution. It is believed that mainly tissue accumulation is responsible for the large volume of distribution of HCQ. Current literature indicates that tissue concentrations of HCQ are well above the plasma concentrations. The concentrations of the HCQ in the lungs might be 200-700 times greater than the plasma concentrations. HCQ accumulates in great amounts in liver, kidneys, lungs and other organs or tissues ${ }^{2,3,16-18}$. Most likely, this property of HCQ provides effective viral inhibiting drug concentrations in tissues ${ }^{1-3}$. In addition, since HCQ is a weak base, it accumulates in acidic compartments in the body mainly in lysosomes (and inflamed tissues) 1,2,7,19-23 which might constitute the majority of the tissue concentration of the drug that might also enable HCQ to block SARS-CoV-2 virus replication ${ }^{1-3,7}$. HCQ could 
be transported into tissues by lymphocytes, leukocytes or monocytes since HCQ can also accumulate in these cells ${ }^{16,19,24}$. The plasma concentrations of $\mathrm{HCQ}$ are well below the inhibitory levels of SARS-CoV-2 virus. The elimination half-life of HCQ is shown to be very long which might mainly be attributed to slow releasing of HCQ from the drug-accumulated tissues into the blood ${ }^{2,16-18}$. The bioavailability of the HCQ might be variable among patients ${ }^{18,20-23}$.

\section{Background of Hypothesis}

Currently, HCQ is used mainly for the treatment of rheumatic diseases. In the context of rheumatology, HCQ has a gradual onset of action that is HCQ is a slow acting anti rheumatic drug and, it may take several weeks to show its action however full therapeutic efficacy may be seen after a few to several months of therapy ${ }^{19-23}$. Steady state drug levels in plasma can be achieved using regular daily HCQ doses after several weeks ${ }^{20,23}$. This is mainly determined by the complex interaction among absorption, distribution, metabolism and elimination of HCQ. Since HCQ have a large volume of distribution, tissue accumulation might be considered as one of the major determinants of these factors. Thus the delayed effect of HCQ in rheumatic diseases might be mainly attributed to its slow accumulation in the tissues ${ }^{19-23}$. In addition, in an article, in which the toxicity and pharmacokinetics of HCQ was evaluated, by administration of $40 \mathrm{mg} / \mathrm{kg} /$ day HCQ orally (by stomach tube), six days a week for three months in albino rats, it was extrapolated from the data that HCQ might gradually accumulate in tissues and the accumulation of HCQ might be rapid for the first 2 weeks however it might also show some further accumulation during 3th month ${ }^{25}$. On the other hand, in another study, by using a simulation method, it was suggested that HCQ might accumulate in lungs rapidly after a loading dose and subsequent maintenance doses ${ }^{3}$ however this suggestion does not seem accordant with the 
literature $\mathrm{e}^{19-23,25}$. Since HCQ might accumulate in tissues gradually in animals ${ }^{25}$, the time to reach steady state HCQ levels in plasma might be several weeks in humans ${ }^{20,23}$ and, HCQ has a gradual onset of action in the treatment of rheumatic diseases ${ }^{19-23}$, it might be proposed that gradual onset of action of HCQ might be valid for the treatment of Covid-19 pneumonia patients, thus a considerably long time might be needed for HCQ to achieve therapeutic drug concentrations in lungs (figure-1), that's why starting HCQ therapy as early as possible seems logical.

Small thrombi, vascular injury, interstitial fibrosis, vascular wall thickening, lumen stenosis and occlusion, diffuse alveolar damage, thickened alveolar capillaries, edema, fibrin thrombi in capillaries and small vessels, and inflammatory cells in diseased areas of the lungs might be seen in Covid-19 pneumonia patients ${ }^{26,27}$. According to histopathological findings, some pulmonary capillaries and some small vessels seem involved (partially) as wall thickening, fibrin thrombi, thrombi, vascular stenosis and occlusion in Covid-19 pneumonia. These abnormalities might cause poor perfusion of diseased lung areas ${ }^{26-29}$. Hypoxic pulmonary vasoconstriction might also contribute to reduced pulmonary perfusion ${ }^{28}$. Reduced perfusion, edema and somewhat distorted architecture of lung tissue might cause reduced drug delivery in these pneumonic areas. Eventually drug concentrations in the pneumonic areas of the lungs might be reduced even though $\mathrm{HCQ}$ is prone to accumulate in (acidic) inflamed tissues due to its weak base property ${ }^{1,2,19-23}$. Since one of the main suggested mechanisms of Covid-19 pneumonia is the attack of SARS-CoV-2 virus to ACE2 receptors on the surface of type- 2 pneumoyctes ${ }^{7,30}$ less HCQ arrival and accumulation in and around these cells might result in inefficient drug concentrations and treatment failure.

Considering reduced drug concentrations in the pneumonic areas of the lungs together with HCQ's large volume of distribution, 
its huge uptake by the tissues, in the context of Covid-19 pneumonia particularly by the lungs, it might be supposed that in a given HCQ dose, the amount of HCQ in healthy lungs might not be comparable to the amount of HCQ in the lungs with pneumonia. That is, it might be proposed that the amount of HCQ could be less in the diseased lungs than in the healthy lungs (figure-2). Suggested gradual HCQ accumulation in the lungs (figure-1) might also contribute to augmentation of the difference between the concentrations of healthy and diseased lungs since the speed of the accumulation of HCQ in the diseased lungs might be lower than the speed of the progression of the pneumonia ${ }^{31,34}$. According to the current literature, Covid-19 pneumonia is usually presented with bilateral patchy ground glass opacities and some consolidations ranging from minimal to extensive involvement of the lungs in chest CT scans ${ }^{31-34}$. The more involvement of the lungs with Covid-19 pneumonia might be the less HCQ concentrations in pneumonic areas (figure-2). Thus, treatment of advanced Covid-19 pneumonia patients with HCQ might result in significant sub-therapeutic drug concentrations in the lungs, which might cause treatment failures.

It is also possible that patients having minimal or limited pulmonary involvement might have less viral loads than patients having extensive lung involvement ${ }^{35}$. This situation could also be valid for the proposed cytokine storm ${ }^{1-3,36}$ in Covid-19 pneumonia patients that might also have strong detrimental effects ${ }^{36}$ on disease progression which could be prevented by HCQ since HCQ might also have beneficial immune modulatory effects ${ }^{1-3,19-22}$. However, as explained above, patients having extensive lung involvement might have less HCQ concentrations in their lungs than patients having limited lung involvement (figure-2), which could possibly associated with some degree of treatment failure in patients with extensive Covid-19 pneumonia; that could also be translated as that $\mathrm{HCQ}$ might not be effective in treatment of 
advanced Covid-19 pneumonia cases considering together with HCQ's suggested gradual onset of action.

In addition, since there might be inter individual bioavailability differences for $\mathrm{HCQ}^{18,20-23}$, treatment failures might be inevitable in patients with low bioavailability and having advanced disease. Furthermore, since the actual therapeutic dose of HCQ for the treatment of Covid-19 pneumonia is not known and HCQ doses in use were generally extrapolated from in vivo or in vitro studies, using current $\mathrm{HCQ}$ doses might have the possibility of resulting in borderline or sub-therapeutic HCQ concentrations in lungs $2,3,6,7,16,17,25$. This might also be an important factor for the proposed treatment failure in patients with advanced Covid-19 pneumonia ${ }^{6,7}$.

Combining above data and assumptions, using HCQ in Covid19 prophylaxis seems logical, since providing enough accumulation of HCQ in the healthy lungs, before the arrival of the SARS-CoV-2 virus and before the lung tissue might become unsuitable for drug uptake due to pneumonia, might prevent Covid-19 pneumonia. In accordance with this hypothesis that in an experimental zika virus infection study in mice ${ }^{37}$, chloroquine (CQ) was shown to be able to prevent zika virus induced mortality when it was used shortly after the infection ( 0 or 12 hours) however it did not show any antiviral effect when used after 24 hours of infection ${ }^{37}$. In another study in mice $^{38}, 50 \mathrm{mg} / \mathrm{kg} /$ day CQ administration was made for 2 days before zika virus infection and the same dosage for 5 days after the beginning of the infection, and $5 \mathrm{mg} / \mathrm{kg} /$ day until end of the experimental procedure. Control mice were administered only drinking water. It was shown that $\mathrm{CQ}$ provided a significantly longer lifespan and survival for zika virus infected mice and alleviated zika virus caused weight $\operatorname{loss}^{38}$. In small studies with Covid-19 patients, it was also shown that HCQ treatment might be successful in treating mild and moderate Covid-19 cases $^{4,5}$ whereas there is no 
study available showing a treatment success in severe or critical pneumonia cases for now.

\section{Summary of The Background of The Hypothesis}

1-Gradual onset of action; since HCQ might accumulate in tissues gradually in animals ${ }^{25}$ and, the time to reach steady state HCQ levels in plasma might be several weeks in humans ${ }^{20,23}$, a considerably long time might be necessary to reach therapeutic drug concentrations in the lungs of the patients with Covid-19 pneumonia.

2-Pneumonia itself might be an obstacle to reach efficient HCQ concentrations in pneumonic areas due to poor perfusion, edema and somewhat architectural distortion of the pneumonic areas $^{26-29}$

3-In addition to poor perfusion, edema and somewhat architectural distortion of the pneumonic areas, existence of extensive disease also might contribute to poor drug delivery in pneumonic areas; since pneumonia is a dynamic and progressive phenomenon and, HCQ might accumulate in lungs gradually, there might not be enough time to reach therapeutic HCQ concentrations in the lungs of the patients with extensive Covid-19 pneumonia after beginning HCQ treatment.

4-Viral load together with cytokine storm might be another factor for treatment failure in advanced disease; the more extension of pneumonia might be associated with the more viral load and the more cytokine storm ${ }^{1-3,35,36}$, however as mentioned above, the more involvement of the lungs with Covid-19 pneumonia might be the less HCQ concentrations in pneumonic areas.

\section{Hypothesis}

1-Since HCQ might accumulate in tissues gradually in animals ${ }^{25}$ and, the time to reach steady state HCQ levels in plasma 
might be several weeks in humans ${ }^{20,23}$ and, HCQ has a gradual onset of action in the treatment of rheumatic diseases ${ }^{19-23}$, which might also be valid for the treatment of Covid-19 pneumonia and, patients having extensive lung involvement might have more viral loads in their lungs than patients having limited lung involvement ${ }^{35}$ and, patients having extensive lung involvement might have significant sub-therapeutic HCQ concentrations in their lungs, treatment of advanced Covid-19 pneumonia patients with HCQ might result in treatment failure. On the contrary, patients with mild or moderate Covid-19 pneumonia might have therapeutic HCQ concentrations in their lungs that might result in treatment success.

2-Since HCQ might accumulate in tissues gradually in animals $^{25}$ and, the time to reach steady state HCQ levels in plasma might be several weeks in humans ${ }^{20,23}$ and, HCQ has a gradual onset of action in the treatment of rheumatic diseases ${ }^{19-23}$, which might also be valid for the treatment of Covid-19 pneumonia and, there might be a likelihood of sub-therapeutic HCQ concentrations in pneumonic areas of the lungs, beginning the administration of the HCQ before the disease onset, that could be also translated as prophylaxis, seems logical. That is, providing enough accumulation of HCQ to achieve therapeutic/protective drug concentrations in healthy lungs, before the arrival of the SARS-CoV-2 virus, might prevent Covid-19 pneumonia.

If the ongoing HCQ clinical trials show promising results at least in the treatment of mild and moderate Covid-19 pneumonia patients, HCQ might be proposed as a suitable candidate for a prophylactic drug in addition to being a therapeutic drug candidate for Covid-19 pneumonia. 


\section{Frequency of Side Effects of HCQ; Is it Really Harmful in} The Context of Treatment or Prophylaxis of Covid-19 Disease?

Currently, HCQ is used mainly for the treatment of rheumatic diseases. Hundreds of thousands of patients or more use HCQ worldwide for these diseases. Even though HCQ can cause many side effects, retinopathy was consistently investigated or reported according to a PubMed search (Accessed 16 April 2020) with the words (in the title) hydroxychloroquine and retinopathy results). Some of the other side effects of HCQ searched and listed on PubMed (in the title) are hydroxychloroquine and cardiomyopathy (22 results), hydroxychloroquine and porphyria (12 results), hydroxychloroquine and psoriasis (11 results), hydroxychloroquine and heart ( 8 results) hydroxychloroquine and cardiac (7 results), hydroxychloroquine and QT (3 results), hydroxychloroquine and hypoglycemia (2 results), hydroxychloroquine and psychosis (2 results) hydroxychloroquine and depression (1 result), and hydroxychloroquine and side effects (4 results) respectively. These results suggest that the side effects other than retinopathy are very rare or not necessarily reported or they are underestimated or they are not seen as frequent as what should be theoretically expected or some combinations of the aforementioned reasons. Retinopathy is a significant side effect however it might appear after consuming daily HCQ doses regularly for a very long period of time, which is not absolutely comparable to the duration of treatment for Covid-19 patients. However no drug is innocent, HCQ also has several significant side effects other than retinopathy as mentioned above, such as QT prolongation, cardiomyopathy, hypoglycemia, neuro-psychiatric side effects, aggravation of psoriasis or porphyria ${ }^{18,21-23}$. QT prolongation is considered as a potentially life-threatening side effect of HCQ however there are only three articles listed after the PubMed search 
with the words (in the title) hydroxychloroquine and QT. Even though conclusive evidence of cardiotoxicitiy caused by HCQ might not be available from the current literature ${ }^{22}$ since it is a potentially life-threatening event and also combined use of certain drugs with HCQ theoretically increase drug toxicity ${ }^{18,22}$, every single person with heart disease or prone to QT prolongation should be examined in detail and carefully monitored before, during and after HCQ therapy. Persons having retinopathy should not use HCQ. In addition, risk and benefit ratio should be determined for every single person before using $\mathrm{HCQ}^{18,22}$.

\section{Conclusion}

If the ongoing $\mathrm{HCQ}$ clinical trials show promising results at least in the treatment of mild and moderate covid-19 pneumonia patients, considering the risk and benefit ratio, in addition to being a candidate for the treatment, HCQ could be a suitable candidate for the prophylaxis of Covid-19 pneumonia at least until a new vaccine or a confirmed new prophylactic drug is available. Furthermore, side effects of HCQ should be re-evaluated in the context of Covid-19 pneumonia prophylaxis and treatment.

The purpose of this paper is only to try to bring a new perspective on the role of HCQ in the treatment or in the prophylaxis of Covid-19 pneumonia. The purpose of this paper is not to recommend using or not using HCQ for the treatment or for the prophylaxis of Covid-19 pneumonia. This paper proposes only hypotheses, which need further researches to be confirmed.

Transparency Declarations: None to declare 


\section{REFERENCES}

1 Zhou D, Dai SM, Tong Q. COVID-19: a recommendation to examine the effect of hydroxychloroquine in preventing infection and progression. J Antimicrob Chemother. 2020 20. pii: dkaa114. DOI: $10.1093 / \mathrm{jac} / \mathrm{dkaa} 114$

2 Liu J, Cao R, Xu M, et al. Hydroxychloroquine, a less toxic derivative of chloroquine, is effective in inhibiting SARS-CoV-2 infection in vitro. Cell Discov. 2020; 18;6:16. DOI:10.1038/s41421020-0156-0

3 Yao X, Ye F, Zhang M, Cui C, et al. In Vitro Antiviral Activity and Projection of Optimized Dosing Design of Hydroxychloroquine for the Treatment of Severe Acute Respiratory Syndrome Coronavirus 2 (SARS-CoV-2). Clin Infect Dis. 2020 9. pii: ciaa237. DOI: $10.1093 /$ cid/ciaa237

4 Gautret et al. (2020) Hydroxychloroquine and azithromycin as a treatment of COVID-19: results of an open-label non-randomized

clinical trial. International Journal of Antimicrobial Agents - In Press 17 March 2020 - DOI : 10.1016/j.ijantimicag.2020.105949

5 Chen Z, Hu J, Zhang Z, et al. Efficacy of hydroxychloroquine in patients with COVID-19: results of a randomized clinical trial. Version 2. medRxiv 2020.03.22.20040758. [Preprint.] doi: $10.1101 / 2020.03 .22 .20040758$

6 Perinel S, Launay M, Botelho-Nevers É, et al. Towards Optimization of Hydroxychloroquine Dosing in Intensive Care Unit COVID-19 Patients. Clin Infect Dis. 2020 Apr 7. pii: ciaa394. doi: 10.1093/cid/ciaa394.

7 Arnold SLM, Buckner F. Hydroxychloroquine for treatment of SARS-CoV-2 infection? Improving our confidence in a model-based approach to dose selection. Clinical and Translational Science https://doi.org/10.1111/cts.12797 
8 COVID 19: Interim Guidance on Management Pending Empirical Evidence. From an American Thoracic Society-led International Task Force $\quad$ https://www.thoracic.org/covid/covid-19-guidance.pdf (Accessed 16 April 2020)

9 Ferner RE, Aronson JK. Chloroquine and hydroxychloroquine in covid-19. BMJ. 2020 Apr 8;369:m1432. doi: 10.1136/bmj.m1432.

10 https://covid19bilgi.saglik.gov.tr/depo/rehberler/COVID19_Rehberi.pdf?type=file (Accessed 11 May 2020)

11 https://epidemio.wiv-isp.be/ID/Documents/Covid19/COVID19_InterimGuidelines_Treatment_ENG.pdf (Accessed 11 May 2020) 12 https://www.mohfw.gov.in/pdf/AdvisoryontheuseofHydroxychlor oquinasprophylaxisforSARSCoV2infection.pdf (Accessed 11 May 2020)

13 Kaushik N, Subramani C, Anang S, et al. Zinc Salts Block Hepatitis E Virus Replication by Inhibiting the Activity of Viral RNADependent RNA Polymerase.J Virol. 2017 Oct 13;91(21). pii: e00754-17. doi: 10.1128/JVI.00754-17

14 Suara RO, Crowe JE Jr. Effect of zinc salts on respiratory syncytial virus replication. Antimicrob Agents Chemother. 2004 Mar;48(3): 783-90.

15 Derwand R, MD, Scholz M. Does zinc supplementation enhance the clinical efficacy of chloroquine/hydroxychloroquine to win todays battle against COVID-19? Preprints (www.preprints.org) doi:10.20944/preprints202004.0124.v1

16 Popert AJ. Chloroquine: a review. Rheumatol Rehabil. 1976; 15(3):235-8.

17 McChesney EW, Banks WF Jr, Fabian RJ. Tissue distribution of chloroquine, hydroxychloroquine, and desethylchloroquine in the rat. Toxicol Appl Pharmacol. 1967; 10(3):501-13. 
18 PLAQUENIL® HYDROXYCHLOROQUINE SULFATE TABLETS, USP https://www.accessdata.fda.gov/drugsatfda_docs/label/2017/00976 8s037s045s047lbl.pdf

19 Abarientos C, Sperber K, Shapiro DL, et al. Hydroxychloroquine in systemic lupus erythematosus and rheumatoid arthritis and its safety in pregnancy. Expert Opin Drug Saf. 2011 Sep;10(5):70514. doi: 10.1517/14740338.2011.566555.

20 Tett SE. Clinical pharmacokinetics of slow-acting antirheumatic drugs. Clin Pharmacokinet. 1993 Nov;25(5):392-407.

21 Haładyj E, Sikora M, Felis-Giemza A, Olesińska M. Antimalarials - are they effective and safe in rheumatic diseases? Reumatologia. 2018;56(3):164-173. doi: 10.5114/reum.2018.76904.

22 Schrezenmeier E, Dörner T. Mechanisms of action of hydroxychloroquine and chloroquine: implications for rheumatology. Nat Rev Rheumatol. 2020 Mar;16(3):155-166. doi: 10.1038/s41584-020-0372-x.

23 Shukla AM, Wagle Shukla A. Expanding horizons for clinical applications of chloroquine, hydroxychloroquine, and related structural analogues. Drugs Context. 2019 Nov 25;8. pii: 2019-9-1. doi: 10.7573/dic.2019-9-1. eCollection 2019.

24 French JK, Hurst NP, O'Donnell ML, et al. Uptake of chloroquine and hydroxychloroquine by human blood leucocytes in vitro: relation to cellular concentrations during antirheumatic therapy. Ann Rheum Dis. 1987; 46(1):42-5

25 McChesney EW. Animal toxicity and pharmacokinetics of hydroxychloroquine sulfate. Am J Med. 1983 Jul 18;75(1A):11-8.

26 Luo, W.; Yu, H.; Gou, J.; Li, X.; Sun, Y.; Li, J.; Liu, L. Clinical Pathology of Critical Patient with Novel Coronavirus Pneumonia (COVID-19). Preprints 2020, 2020020407

27 Fox SE,Akmatbekov A, Harbert JL, et al. Pulmonary and Cardiac Pathology in Covid-19: The First Autopsy Series from New Orleans. medRxiv. doi: https://doi.org/10.1101/2020.04.06.20050575 
28 Yuan A, Yang PC, Lee $L$, et al. Reactive pulmonary artery vasoconstriction in pulmonary consolidation evaluated by color Doppler ultrasonography. Ultrasound Med Biol. 2000; 26(1):49-56.

29 Björk L, McNeil BJ. Blood flow in pulmonary and bronchial arteries in acute experimental pneumonia and pulmonary embolism. Acta Radiol Diagn (Stockh). 1977 Jul;18(4):393-9.

30 Hakim ST, Boyd FT, Soto JA. The Pathophysiology of Virulence of the COVID-19. Preprints (www.preprints.org) I NOT PEERREVIEWED I Posted: 7 April 2020 doi: 10.20944/preprints202004.0077.v1

31. Pan $F, Y e T$, Sun $P$, et al. Time Course of Lung Changes On Chest CT During Recovery From 2019 Novel Coronavirus (COVID19) Pneumonia. Radiology. 2020 Feb 13:200370. doi: 10.1148/radiol.2020200370. [Epub ahead of print]

32 Shi $H$, Han X, Jiang N, Shi $H$, Han X, Jiang $N$, et al. Radiological findings from 81 patients with COVID-19 pneumonia in Wuhan, China: a descriptive study. Lancet Infect Dis. 2020 24. pii: S14733099(20)30086-4.

33 Zhang $R$, Ouyang $H$, Fu $L$, et al. CT features of SARS-CoV-2 pneumonia according to clinical presentation: a retrospective analysis of 120 consecutive patients from Wuhan city.Eur Radiol. 2020 Apr 11. doi: 10.1007/s00330-020-06854-1.

34 Liu KC, Xu P, Lv WF, et al. CT manifestations of coronavirus disease-2019: A retrospective analysis of 73 cases by disease severity. Eur J Radiol. 2020 Mar 12;126:108941. doi: 10.1016/j.ejrad.2020.108941.

35 Oh MD, Park WB, Choe PG, et al. Viral Load Kinetics of MERS Coronavirus Infection. N Engl J Med. 2016 Sep 29;375(13):1303-5. doi: $10.1056 /$ NEJMc1511695.

36 Shi $Y$, Wang $Y$, Shao $C$, et al. COVID-19 infection: the perspectives on immune responses. Cell Death Differ. 2020 Mar 23. doi: $10.1038 / s 41418-020-0530-3$. 
37 Chunfeng Li, Xingliang Zhu, Xue Ji, et al. Chloroquine, a FDAapproved Drug, Prevents Zika Virus Infection and its Associated Congenital Microcephaly in Mice EBioMedicine. 2017 Oct; 24: 189194. Published online 2017 Sep 28. doi: 10.1016/j.ebiom.2017.09.034

38 Shiryaev SA, Mesci P, Pinto A, et al. Repurposing of the antimalaria drug chloroquine for Zika Virus treatment and prophylaxis. Sci Rep. 2017 Nov 17;7(1):15771. doi: 10.1038/s41598-01715467-6. 


\section{LEGENDS OF FIGURES}

Figure-1 Schematic representation of hypothetical gradual HCQ accumulation in lungs of humans (each circle represents HCQ). Since HCQ might accumulate in tissues gradually in animals ${ }^{25}$, the time to reach steady state HCQ levels in plasma might be several weeks in humans $s^{20,23}$ and, HCQ has a gradual onset of action in the treatment of rheumatic diseases ${ }^{19-23}$, it might be proposed that gradual onset of action of HCQ might be valid for the treatment of Covid-19 pneumonia patients, thus a considerably long time might be needed for HCQ to achieve therapeutic drug concentrations in lungs.

Figure-2: Schematic representation of Covid-19 pneumonia, hypothetical pneumonic involvement varying in severity (from limited to extended disease) and, HCQ therapy. Reduced perfusion, somewhat distorted architecture of lung tissue, edema and suggested gradual accumulation of $\mathrm{HCQ}$ in lung tissue 19-23,25,26-29 might cause reduced HCQ delivery and HCQ concentrations in pneumonic areas. The more involvement of the lungs with Covid-19 pneumonia might be the less $\mathrm{HCQ}$ concentrations in pneumonic areas. (Each circle represents $\mathrm{HCQ}$, colored areas indicate pneumonic involvement) 


\section{FIGURES}
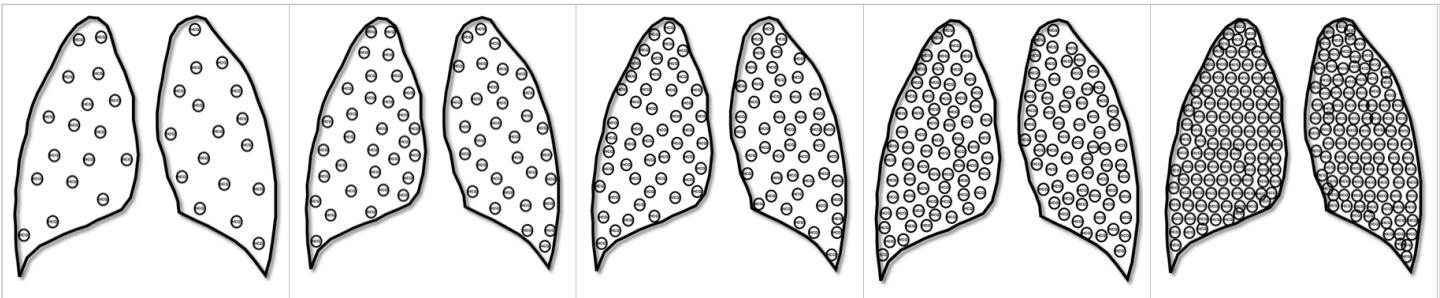

Time: Days (or weeks)

Figure-1

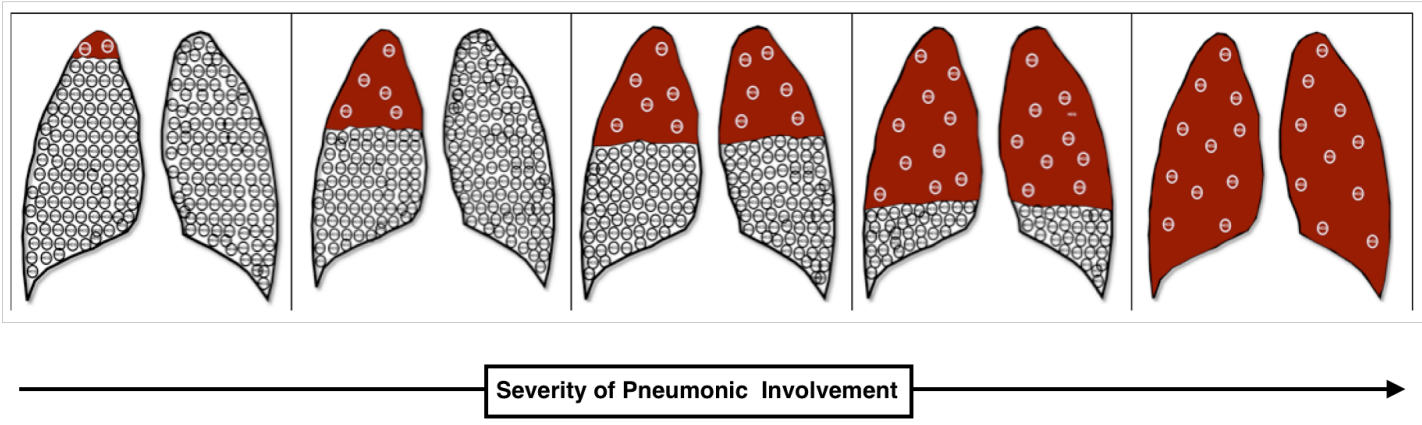

Figure-2 